\title{
On Efficient Application of Implicit Runge-Kutta Methods to Large-Scale Systems of Index 1 Differential-Algebraic Equations ${ }^{\star}$
}

\author{
Gennady Yu. Kulikov ${ }^{1}$ and Alexandra A. Korneva ${ }^{1}$ \\ Ulyanovsk State University, L. Tolstoy Str. 42, 432700 Ulyanovsk, Russia
}

\begin{abstract}
In the paper we study how to integrate numerically largescale systems of semi-explicit index 1 differential-algebraic equations by implicit Runge-Kutta methods. In this case, if Newton-type iterations are applied to the discrete problems we need to solve high dimension linear systems with sparse coefficient matrices. Therefore we develop an effective way for packing such matrices of coefficients and derive special Gaussian elimination for parallel factorization of nonzero blocks of the matrices. As a result, we produce a new efficient procedure to solve linear systems arising from application of Newton iterations to the discretizations of large-scale index 1 differential-algebraic equations obtained by implicit Runge-Kutta methods. Numerical tests support theoretical results of the paper.
\end{abstract}

\section{Introduction}

In this paper we deal with index 1 differential-algebraic systems of the form

$$
\begin{gathered}
x^{\prime}(t)=g(x(t), y(t)), \\
y(t)=f(x(t), y(t)), \\
x(0)=x^{0}, y(0)=y^{0} .
\end{gathered}
$$

where $t \in[0, T], x(t) \in \mathbf{R}^{m}, y(t) \in \mathbf{R}^{n}, g: D \subset \mathbf{R}^{m+n} \rightarrow \mathbf{R}^{m}, f: D \subset \mathbf{R}^{m+n} \rightarrow$ $\mathbf{R}^{n}$, and where initial conditions (1c) are consistent; i. e., $y^{0}=f\left(x^{0}, y^{0}\right)$. Note that we consider only autonomous systems because any nonautonomous system may be converted to an autonomous one by introducing a new independent variable.

To solve problem (1) numerically, we apply an $l$-stage implicit Runge-Kutta (RK) method given by the table

$$
\frac{c \mid A}{b^{T}},
$$

\footnotetext{
* This work was supported in part by the Russian Foundation of the Basic Research (grants No. 01-01-00066 and No. 00-01-00197).
} 
where $A$ is a real matrix of dimension $l \times l, b$ and $c$ are real vectors of dimension $l$, to problem (1) and obtain the following discrete problem:

$$
\begin{gathered}
x_{k i}=x_{k}+\tau \sum_{j=1}^{l} a_{i j} g\left(x_{k j}, y_{k j}\right), \\
y_{k i}=f\left(x_{k i}, y_{k i}\right), \quad i=1,2, \ldots, l, \\
x_{k+1}=x_{k}+\tau \sum_{i=1}^{l} b_{i} g\left(x_{k i}, y_{k i}\right), \\
y_{k+1}=f\left(x_{k+1}, y_{k+1}\right), \quad k=0,1, \ldots, K-1, \\
x_{0}=x^{0}, \quad y_{0}=y^{0}
\end{gathered}
$$

where $\tau$ is a stepsize. Algebraic system (2) is solved then by an iterative method. Usually the iterative process is taken in the form of simple or Newton-type iterations with trivial (or nontrivial) predictor [1], 4], 7], 8], [10], 12].

Newton (or modified Newton) iterations are more preferable for solving differential-algebraic equations (1) than simple ones. First, RK method (2) is convergent in the case of simple iterations only if the infinite norm of the Jacobian of the right-hand part of (1b) is bounded on a convex set containing the exact solution of the original problem by a constant $d<1$ (see theorem 2 in 8 ] and theorem 3 in [10]). In the case of Newton-type iterations it is enough to satisfy the implicit function theorem for algebraic part (1b) in order to obtain convergent numerical methods (see theorems 3, 4 in 8 and theorems 1, 2 in 10 ). The last condition holds obviously for any sufficiently smooth differential-algebraic system (1) of index 1. This follows from 2], 3] and [6. Second, when using Newton (full or modified) method we can limit ourselves by a less number of iterations to attain the order of the underlying RK formula. Moreover, if we apply an RK method of sufficiently high stage order with appropriate nontrivial predictor to problem (1) then we can carry out only 2 iterations per time point to get the maximum order convergence [10].

The only drawback of Newton iterations is severe requirements on RAM and CPU time caused by the increasing dimension of discrete problem (2) in $l$ times when an $l$-stage implicit RK method is used. Thus, it is necessary to solve linear systems of dimension $(m+n) l$ many times during the integration. Therefore the basic problem is how to simplify the numerical solving of the linear systems with coefficient matrices of the special form arising from the application of Newton methods (full or modified) to differential-algebraic system (1).

In the paper we use the special structure of the matrices mentioned above to obtain a modification of Gaussian elimination. This modification allows RAM and CPU time to be significantly reduced in the numerical integration of problem (1) by an implicit RK method. We give the estimates of such reduction both in theory and in practice.

Finally, we study how to integrate numerically large-scale systems of differential-algebraic equations of index 1 . In this case we need to solve high dimension 
linear systems with sparse coefficient matrices. We develop firstly an effective way for packing such matrices of coefficients. After that we derive special Gaussian elimination for parallel factorization of nonzero blocks of the matrices. Thus, we produce a new efficient procedure to solve linear systems arising from application of Newton iterations to the discretizations of large-scale index 1 differentialalgebraic systems obtained by implicit RK methods. We also give numerical tests which support the theoretical results.

\section{Efficient Implementation of Iterative Runge-Kutta Methods}

In the preceding section we have shown that Newton iterations are effective to solve semi-explicit index 1 differential-algebraic equations numerically. The basic part of the Newton method applied to discrete problem (2) consists of solving linear systems of the form

$$
\partial \bar{F}_{k}^{\tau}\left(Z_{k+1}^{i-1}\right)\left(Z_{k+1}^{i-1}-Z_{k+1}^{i}\right)=\bar{F}_{k}^{\tau} Z_{k+1}^{i-1}
$$

where lower indices mean time points, and upper ones denote iterations. Here $Z_{k+1} \stackrel{\text { def }}{=}\left(\left(z_{k 1}\right)^{T}, \ldots,\left(z_{k, l-1}\right)^{T},\left(z_{k l}\right)^{T}\right)^{T} \in \mathbf{R}^{(m+n) l}$, where the vector $z_{k j} \stackrel{\text { def }}{=}$ $\left(\left(x_{k j}\right)^{T},\left(y_{k j}\right)^{T}\right)^{T} \in \mathbf{R}^{m+n}, j=1,2, \ldots, l$, unites components of the $j$-th stage value of $l$-stage RK formula (2). The mapping $\bar{F}_{k}^{\tau}$ is the nontrivial part of discrete problem (2) for computing the stage values $Z_{k+1}$, and $\partial \bar{F}_{k}^{\tau}\left(Z_{k+1}^{i-1}\right)$ denotes the Jacobian of the mapping $\bar{F}_{k}^{\tau}$ evaluated at the point $Z_{k+1}^{i-1}$.

It is easy to see that the matrix $\partial \bar{F}_{k}^{\tau}\left(Z_{k+1}^{i-1}\right)$ has the block structure

$$
\partial \bar{F}_{k}^{\tau}(Z) \stackrel{\text { def }}{=}\left(\begin{array}{c}
\partial \bar{F}_{k}^{\tau}(Z)_{1} \\
\partial \bar{F}_{k}^{\tau}(Z)_{2} \\
\vdots \\
\partial \bar{F}_{k}^{\tau}(Z)_{l}
\end{array}\right)
$$

where each block $\partial \bar{F}_{k}^{\tau}(Z)_{j}, j=1,2, \ldots, l$, is an $(m+n) \times(m+n) l$-matrix of the form

$$
\left(\begin{array}{cccccccccccc}
O(\tau) & \cdots & O(\tau) & 1+O(\tau) & \cdots & O(\tau) & O(\tau) & \cdots & O(\tau) & O(\tau) & \cdots & O(\tau) \\
\vdots & \ddots & \vdots & \vdots & \ddots & \vdots & \vdots & \ddots & \vdots & \vdots & \ddots & \vdots \\
O(\tau) & \cdots & O(\tau) & O(\tau) & \cdots & 1+O(\tau) & O(\tau) & \cdots & O(\tau) & O(\tau) & \cdots & O(\tau) \\
0 & \cdots & 0 & z & \cdots & z & z & \cdots & z & 0 & \cdots & 0 \\
\vdots & \ddots & \vdots & \vdots & \ddots & \vdots & \vdots & \ddots & \vdots & \vdots & \ddots & \vdots \\
0 & \cdots & 0 & \underbrace{z}_{(m+n)(j-1)} & \cdots & z & z & \cdots & z & 0 & \cdots & 0
\end{array}\right\} n
$$

Here $z$ means in general a nontrivial element. 
Having used the structure of matrix (4), Kulikov and Thomsen suggested in [7] to exclude the zero blocks situated below the main diagonal from LUfactorization and forward substitution. That reduces the number of arithmetical operations and, hence, CPU time significantly when linear system (3) is solved. However, the advantage of the new version of Gaussian elimination becomes greater if we have interchanged the $x$-components of the vector $Z_{k+1}$ with the corresponding $y$-components. It means that we have interchanged the first $m$ rows with the last $n$ ones in each submatrix $\partial \bar{F}_{k}^{\tau}(Z)_{j}$. In this case we can exclude all the zero blocks of matrix (4) from the LU-factorization, forward and backward substitutions and solve problem (3) very effectively 9 .

Let us now compare efficiency of all the versions of Gaussian elimination for linear system (3). To do this, we compute and compare the total number of multiplications and divisions for each version when $m=n=2$ and $l=1,2,3,4$.

It is well-known that the ordinary Gauss method requires

$$
\frac{(m+n) l\left(((m+n) l)^{2}+3(m+n) l-1\right)}{3}
$$

operations of multiplication and division (see, for example, 13). The total number of operations for Kulikov and Thomsen's version (Modification I) can be easily obtained from [7]

$$
\begin{gathered}
((m+n) l)^{2}+\frac{((m+n) l-1)(m+n) l(2(m+n) l-1)}{6} \\
+\frac{(m+n) l(m l+n-1)}{2}-\frac{n(m+n)^{2}(l-1) l(2 l-1)}{6} \\
-\frac{n(m+n)(m+n-1) l(l-1)}{4} .
\end{gathered}
$$

The formula

$$
\begin{gathered}
(m+n) l(m l+n)+\frac{(m+n-1)(m+n)(2(m+n)-1) l}{6} \\
+\frac{m(m+n)(2 m+n) l(l-1)}{4}+\frac{m^{2}(m+n)(l-1) l(2 l-1)}{6} \\
+\frac{n(n-1) l}{2}+\frac{m(m-1) l}{2}+m n l
\end{gathered}
$$

gives the number of multiplications and divisions for Kulikov and Korneva's version (Modification II) 9$]$.

Now we substitute $m=n=2$ and $l=1,2,3,4$ in formulas (5)-(7) and obtain Table 1. We see from the table that Modification II is the most preferable to be used even in such a low dimension case. For example, this version of Gaussian 
Table 1. Total numbers of multiplications and divisions when $m=n=2$

\begin{tabular}{|c|c|c|c|}
\hline $\begin{array}{c}\text { Number of } \\
\text { stages, } l\end{array}$ & $\begin{array}{c}\text { Gauss } \\
\text { method }\end{array}$ & $\begin{array}{c}\text { Modification } \\
\text { I }\end{array}$ & $\begin{array}{c}\text { Modification } \\
\text { II }\end{array}$ \\
\hline 1 & 36 & 36 & 36 \\
2 & 232 & 180 & 128 \\
3 & 716 & 496 & 308 \\
4 & 1616 & 1048 & 608 \\
\hline
\end{tabular}

Table 2. RAM (in bytes) needed to store matrix (4) when $m=n=2$

\begin{tabular}{|c|c|c|c|}
\hline $\begin{array}{c}\text { Number of } \\
\text { stages, } l\end{array}$ & $\begin{array}{c}\text { Gauss } \\
\text { method }\end{array}$ & $\begin{array}{c}\text { Modification } \\
\text { I }\end{array}$ & $\begin{array}{c}\text { Modification } \\
\text { II }\end{array}$ \\
\hline 1 & 128 & 128 & 128 \\
2 & 512 & 448 & 384 \\
3 & 1152 & 960 & 768 \\
4 & 2048 & 1664 & 1280 \\
\hline
\end{tabular}

elimination requires roughly three times less operations for 4-stage implicit RK formulas than the ordinary Gauss method. It is also better than Modification I. The advantage of this method will obviously increase for a higher dimension differential-algebraic systems (1).

Except CPU time, it is important to compare RAM needed to store matrix (4). If we assume that the type double is used to store elements of the matrix then Table 2 gives such information. These data also confirm advantage of Modification II over other methods.

\section{Large-Scale Systems}

In Section 2 we presented the efficient way of applying implicit RK methods to problem (1). Unfortunately, it is not enough when we solve large-scale semiexplicit index 1 differential-algebraic systems. As an example, we may take the model of overall regulation of body fluids [5. This model is a problem of the form (1) containing about two hundred variables. Having applied any implicit 3- or 4-stage RK method to the model we encounter the situation when the dimension of discrete problem (2) is too high to solve it by Newton iterations. On the other hand, the Jacobian of a large-scale differential-algebraic system is often a sparse matrix. For instance, the model mentioned above is the case. Thus, we must solve two problems in this section. The first problem is how to pack a matrix of the form (4) excluding all trivial elements. The second one is how to implement Modification II of Gaussian elimination for the packed matrix (4) efficiently. 
First of all we rearrange the variables and define the vectors:

$$
\begin{gathered}
X_{k+1} \stackrel{\text { def }}{=}\left(\left(x_{k 1}\right)^{T}, \ldots,\left(x_{k, l-1}\right)^{T},\left(x_{k l}\right)^{T}\right)^{T} \in \mathbf{R}^{m l}, \\
Y_{k+1} \stackrel{\text { def }}{=}\left(\left(y_{k 1}\right)^{T}, \ldots,\left(y_{k, l-1}\right)^{T},\left(y_{k l}\right)^{T}\right)^{T} \in \mathbf{R}^{n l} .
\end{gathered}
$$

Now $Z_{k+1} \stackrel{\text { def }}{=}\left(\left(X_{k+1}\right)^{T},\left(Y_{k+1}\right)^{T}\right)^{T}$ and matrix (4) has the form

$$
\partial \bar{F}_{k}^{\tau}(Z) \stackrel{\text { def }}{=}\left(\begin{array}{c}
\partial \bar{F}_{k}^{\tau}(Z)^{Y} \\
\partial \bar{F}_{k}^{\tau}(Z)^{X}
\end{array}\right) .
$$

Here each submatrix has also the block structure

$$
\partial \bar{F}_{k}^{\tau}(Z)^{Y} \stackrel{\text { def }}{=}\left(\begin{array}{c}
\partial \bar{F}_{k}^{\tau}(Z)_{1}^{Y} \\
\partial \bar{F}_{k}^{\tau}(Z)_{2}^{Y} \\
\vdots \\
\partial \bar{F}_{k}^{\tau}(Z)_{l}^{Y}
\end{array}\right) \quad \text { and } \quad \partial \bar{F}_{k}^{\tau}(Z)^{X} \stackrel{\text { def }}{=}\left(\begin{array}{c}
\partial \bar{F}_{k}^{\tau}(Z)_{1}^{X} \\
\partial \bar{F}_{k}^{\tau}(Z)_{2}^{X} \\
\vdots \\
\partial \bar{F}_{k}^{\tau}(Z)_{l}^{X}
\end{array}\right)
$$

where

$$
\partial \bar{F}_{k}^{\tau}(Z)_{i}^{Y} \stackrel{\text { def }}{=} \underbrace{\left(\begin{array}{ccccccccccccccc}
0 & \cdots & 0 & z & \cdots & z & 0 & \cdots & 0 & z & \cdots & z & 0 & \cdots & 0 \\
\vdots & \ddots & \vdots & \vdots & \ddots & \vdots & \vdots & \ddots & \vdots & \vdots & \ddots & \vdots & \vdots & \ddots & \vdots \\
0 & \cdots & 0 & z & \cdots & z & 0 & \cdots & 0 & z & \cdots & z & 0 & \cdots & 0
\end{array}\right)}_{n(i-1)} \underbrace{\underbrace{\underbrace{}_{m}}_{n(l-i)+m(i-1)}}_{n} \underbrace{\underbrace{}_{m(l-i)}}_{m}
$$

and

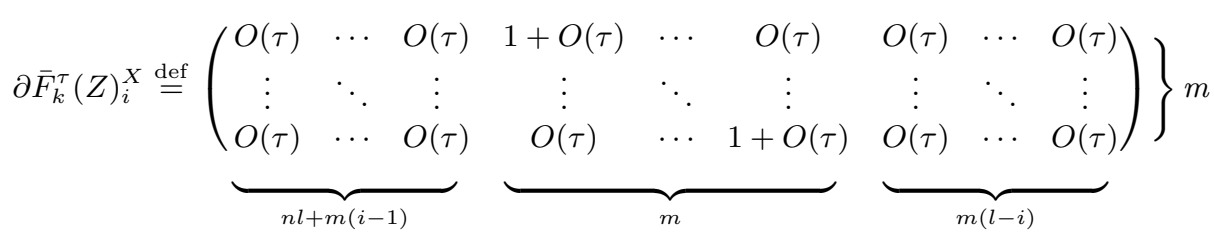

We note that, when solving linear system (3) with matrix (8) by Modification II of Gaussian elimination, LU-factorization of any submatrix $\partial \bar{F}_{k}^{\tau}(Z)_{i}^{Y}$ does not influence the submatrices $\partial \bar{F}_{k}^{\tau}(Z)_{j}^{Y}$ for $j \neq i$. This means that the factorization of the matrix $\partial \bar{F}_{k}^{\tau}(Z)^{Y}$ falls into $l$ independent LU-factorizations of the submatrices $\partial \bar{F}_{k}^{\tau}(Z)_{i}^{Y}, i=1,2, \ldots, l$. Moreover, structures of the similar nonzero blocks of all the submatrices $\partial \bar{F}_{k}^{\tau}(Z)_{i}^{Y}$ (i.e., the number and the places of nonzero elements) coincide, if the stepsize $\tau$ is sufficiently small.

Taking into account the above observation we conclude that the storage of nonzero elements of the matrix $\partial \bar{F}_{k}^{\tau}(Z)^{Y}$ has to give simultaneous access to all elements corresponding components of the stage values with the same subscript. It allows for the parallel factorization of the matrix $\partial \bar{F}_{k}^{\tau}(Z)^{Y}$. To provide this, we will store the matrix $\partial \bar{F}_{k}^{\tau}(Z)^{Y}$ in the form of an array of links to chained lists. Every component of the list representation consists of:

- an $l$ dimension array to store elements of the matrix $\partial \bar{F}_{k}^{\tau}(Z)^{Y}$ corresponding components of the stage values with the same subscript $\left(f_{i j}\right)$; 


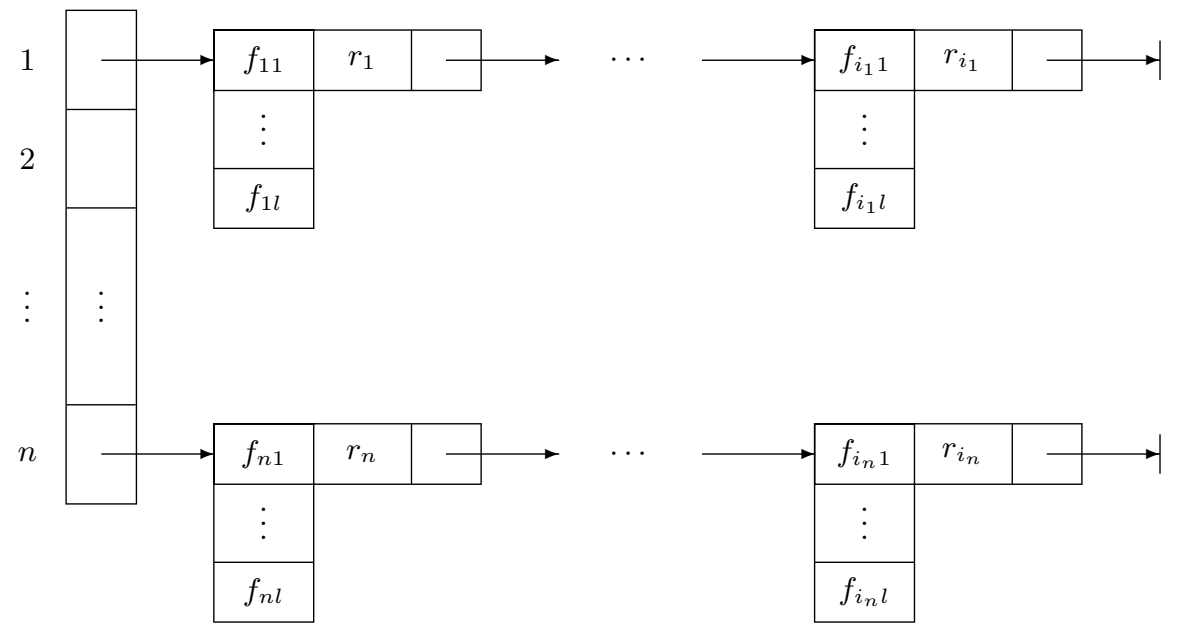

Fig. 1. The storage of nonzero elements of the matrix $\partial \bar{F}_{k}^{\tau}(Z)^{Y}$.

- the subscript of components of the stage values $\left(r_{i}\right)$;

- a link to the next element of the list (or to $n i l$ ).

Since the dimension of the matrix $\partial \bar{F}_{k}^{\tau}(Z)^{Y}$ is equal to $n l$ we need $n$ such lists (see Fig. 1).

To store the matrix $\partial \bar{F}_{k}^{\tau}(Z)^{X}$, we can use any packing appropriate for sparse matrices in a general case because the matrix of coefficients of the RK method may have zero elements and, hence, $\partial \bar{F}_{k}^{\tau}(Z)^{X}$ also contains zero blocks. Fortunately, we will have great advantage by applying only RK methods with dense coefficient matrix $A$ (i.e., $a_{i j} \neq 0, i, j=1,2, \ldots, l$ ) to problem (1). We call such methods dense RK methods. For example, Gauss methods are dense RK methods (see [4]). And there exist no dense methods among explicit RK formulas.

It is easy to see that the matrix $\partial \bar{F}_{k}^{\tau}(Z)^{X}$ can be obtained by pairwise products of elements of the matrices $\tau A$ and $\partial g^{l}(Z)$ where the $m \times(m+n) l$-matrix

$$
\partial g^{l}\left(Z_{k+1}\right) \stackrel{\text { def }}{=}\left(\partial_{y_{k 1}} g\left(z_{k 1}\right) \ldots \partial_{y_{k l}} g\left(z_{k l}\right) \partial_{x_{k 1}} g\left(z_{k 1}\right) \ldots \partial_{x_{k l}} g\left(z_{k l}\right)\right) .
$$

Therefore if we have applied a dense RK formula to problem (1) then all the submatrices $\partial \bar{F}_{k}^{\tau}(Z)_{i}^{X}, i=1,2, \ldots, l$, of the matrix $\partial \bar{F}_{k}^{\tau}(Z)^{X}$ have the same structure, maybe, except the diagonal elements. Moreover, the structure of any submatrix $\partial \bar{F}_{k}^{\tau}(Z)_{i}^{X}$ and the structure of the matrix $\partial g^{l}(Z)$ coincide with the exception mentioned above. Then all nonzero elements of the matrix $\partial \bar{F}_{k}^{\tau}(Z)^{X}$ can be reconstructed by the matrix $\tau A$ and nonzero elements of the matrix $\partial g^{l}(Z)$. Thus, if the stage number $l>1$, and $m, n$ are large, it is better to store two matrices of dimensions $l \times l$ and $m \times(m+n) l$ instead of one matrix of dimension $m l \times(m+n) l$.

Now we discuss Gaussian elimination for system (3) with matrix (8). It was noted earlier that the elimination of variables from system (3) is splitted up 
into two stages. We eliminated the $y$-components, by using the parallel factorization of the submatrices $\partial \bar{F}_{k}^{\tau}(Z)_{i}^{Y}, i=1,2, \ldots, l$, on the first stage. Then we eliminated the $x$-components. The first stage is more important for optimization because the most part of arithmetical operations falls on it. Therefore we give further a way to decrease the number of operations at this stage.

Let us consider the reduced matrix

$$
\left(\begin{array}{c}
\partial \bar{F}_{k}^{\tau}(Z)^{Y} \\
\partial g^{l}(Z)
\end{array}\right)
$$

of dimension $(n l+m) \times(m+n) l$. The next theorem establishes that we can use the reduced matrix (10) instead of the full matrix (8) while eliminating the $y$-components. That is more preferable for us.

Theorem 1. Let a dense l-stage RK formula with coefficient matrix $A$ be used for constructing matrix (8). Then the matrix $\left[\partial \bar{F}_{k}^{\tau}(Z)^{X}\right]^{(\mu)}$ obtained after the $\mu$-th step of Gaussian elimination can be reconstructed uniquely by pairwise products of elements of the matrices $\tau A$ and $\left[\partial g^{l}(Z)\right]^{(\mu)}$ when $0 \leq \mu \leq n l$.

The proof of theorem 1 will appear in [11]. Thus, taking into account this theorem we use the lower dimension matrix (10) on the first $n l$ steps of the Gaussian elimination. After that we reconstruct the matrix $\left[\partial \bar{F}_{k}^{\tau}(Z)^{X}\right]^{(n l)}$ by the matrices $\tau A$ and $\left[\partial g^{l}(Z)\right]^{(n l)}$ and proceed the elimination of the $x$-components of system (3). However, we must remember the parallel factorization of the matrix $\partial \bar{F}_{k}^{\tau}(Z)^{Y}$. By this reason, we have also to store nonzero elements of the matrix $\partial g^{l}(Z)$ given in (9) by the packing suggested for the matrix $\partial \bar{F}_{k}^{\tau}(Z)^{Y}$ (see Fig. 1). The only difference is the number of the chained lists that are necessary to store the matrix $\partial g^{l}(Z)$. In this case we use $m$ such lists.

\section{Numerical Example}

To compare all the versions of Gaussian elimination presented in this paper, we take the model of overall regulation of body fluids mentioned above as a test problem. The version from Section 3 is called further Modification III. To solve the problem in the interval $[0,10]$, we apply Gauss-type implicit RK methods up to order 8 with the stepsize $\tau=1 / 60$ and fulfil two Newton iterations per time point. Table 3 contains execution time (in sec.) for all the versions of Gaussian elimination. Lines in the table mean that the Jacobian of the discrete problem exceeds the available RAM.

This practical example shows that Modification III is the best method to solve linear problems arising in application of implicit RK formulas to large-scale systems of semi-explicit index 1 differential-algebraic equations. Indeed, we see from Figure 2 that the Jacobian of the model of overall regulation of body fluids is a sparse matrix (points mean nonzero elements), and Modification III operates only with the nonzero elements. Also it is important to note that the growth of 


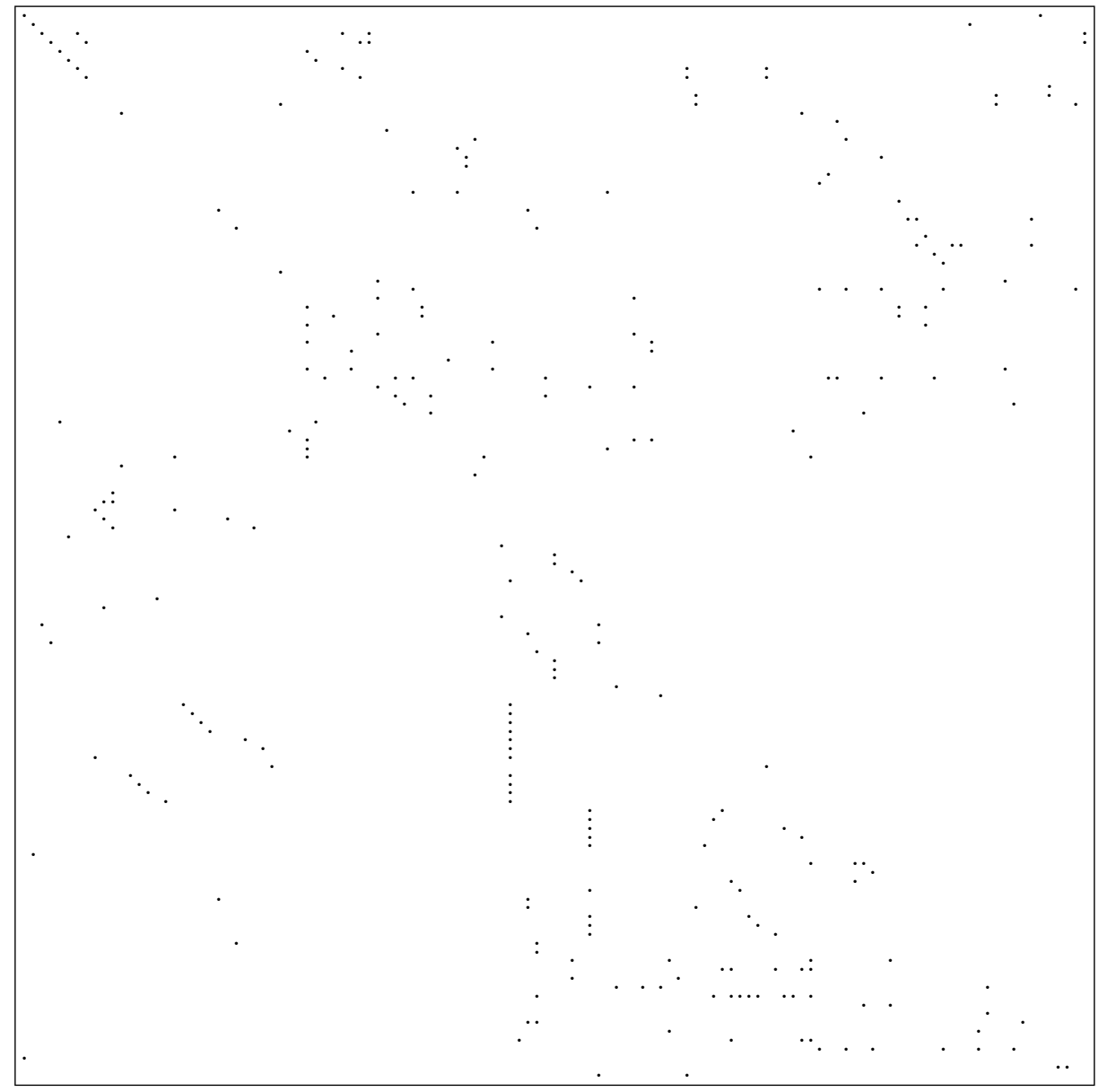

Fig. 2. The structure of the Jacobian of the model of overall regulation of body fluids.

Table 3. Execution time (in sec.) for the processor Intel Pentium 200

\begin{tabular}{|c|c|c|c|c|}
\hline $\begin{array}{c}\text { Number of } \\
\text { stages, } l\end{array}$ & $\begin{array}{c}\text { Gauss } \\
\text { method }\end{array}$ & $\begin{array}{c}\text { Modification } \\
\text { I }\end{array}$ & $\begin{array}{c}\text { Modification } \\
\text { II }\end{array}$ & $\begin{array}{c}\text { Modification } \\
\text { III }\end{array}$ \\
\hline 1 & 1169.85 & 993.05 & 983.77 & 51.80 \\
2 & 9271.15 & 4146.33 & 2551.29 & 215.80 \\
3 & - & - & - & 679.71 \\
4 & - & - & - & 763.90 \\
\hline
\end{tabular}


the execution time decreases with the growth of the number of stages in implicit RK formulas. It is a good result to apply implicit RK formulas of high order in practice.

\section{References}

1. Ascher, U.M., Petzold, L.P.: Computer methods for ordinary differential equations and differential-algebraic equations. SIAM, Philadelphia, 1998

2. Gear, C.W., Petzold, L.R.: ODE methods for the solution of differential/algebraic systems. SIAM J. Numer. Anal. 21 (1984) 716-728

3. Gear, C.W.: Differential-algebraic equations index transformations. SIAM J. Sci. Stat. Comput., 9 (1988) 39-47

4. Hairer, E., Wanner, G.: Solving ordinary differential equations II: Stiff and differential-algebraic problems. Springer-Verlag, Berlin, 1991

5. Ikeda, N., Marumo, F., Shiratare, M., Sato, T.: A model of overall regulation of body fluids. Ann. Biomed. Eng. 7 (1979) 135-166

6. Kulikov, G.Yu.: The numerical solution of the autonomous Cauchy problem with an algebraic relation between the phase variables (non-degenerate case). (in Russian) Vestnik Moskov. Univ. Ser. 1 Mat. Mekh. (1993) No. 3, 6-10; translation in Moscow Univ. Math. Bull. 48 (1993) No. 3, 8-12

7. Kulikov, G.Yu., Thomsen, P.G.: Convergence and implementation of implicit Runge-Kutta methods for DAEs. Technical report 7/1996, IMM, Technical University of Denmark, Lyngby, 1996

8. Kulikov, G.Yu.: Convergence theorems for iterative Runge-Kutta methods with a constant integration step. (in Russian) Zh. Vychisl. Mat. Mat. Fiz. 36 (1996) No. 8, 73-89; translation in Comp. Maths Math. Phys. 36 (1996) No. 8, 1041-1054

9. Kulikov, G.Yu., Korneva, A.A.: On effective implementation of iterative RungeKutta methods for differential-algebraic equations of index 1. (in Russian) In: Basic problems of mathematics and mechanics. 3 (1997), Ulyanovsk State University, Ulyanovsk, 103-112

10. Kulikov, G.Yu.: Numerical solution of the Cauchy problem for a system of differential-algebraic equations with the use of implicit Runge-Kutta methods with nontrivial predictor. (in Russian) Zh. Vychisl. Mat. Mat. Fiz. 38 (1998) No. 1, 6884; translation in Comp. Maths Math. Phys. 38 (1998) No. 1, 64-80

11. Kulikov, G.Yu., Korneva, A.A.: On numerical solution of large-scale systems of index 1 differential-algebraic equations. (in Russian) Fundam. Prikl. Mat. (to appear)

12. Kværnø, A.: The order of Runge-Kutta methods applied to semi-explicit DAEs of index 1, using Newton-type iterations to compute the internal stage values. Technical report 2/1992, Mathematical Sciences Div., Norwegian Institute of Technology, Trondheim, 1992

13. Samarskiy, A.A., Gulin, A.V.: Numerical methods. Nauka, Moscow, 1989 